\title{
Development of innovative embedding procedures for the analyses of paint cross sections in ATR FITR microscopy
}

\author{
S. Prati • G. Sciutto • E. Catelli • A. Ashashina • R. Mazzeo
}

Received: 8 June 2012 /Revised: 12 September 2012 / Accepted: 13 September 2012 /Published online: 6 October 2012

(C) Springer-Verlag Berlin Heidelberg 2012

\begin{abstract}
We report the development of innovative embedding procedures for the analysis of paint cross sections by attenuated total reflection (ATR) Fourier transform IR microscopy. This technique was chosen because it is widely employed for the characterization and spatial location of organic and inorganic components in artistic samples. Moreover, the performance of the technique may be critically affected by sample preparation in terms of surface morphology and the presence of contamination. First, we evaluated the use of $\mathrm{KBr}$ as a barrier to contamination by the embedding synthetic medium. In this way, the sample cross section can be polished by means of a sample holder, which allows a controlled pressure to be applied to the sample, thus improving the reproducibility and quality of the surface cross section. In addition, argon ion milling was used for the polishing of samples embedded in $\mathrm{KBr}$, and provided very promising results in terms of surface planarity and reduction of superficial contamination by KBr. Finally, the use of $\mathrm{NaCl}$ as an alternative to $\mathrm{KBr}$ was proposed thanks to its advantages in terms of hygroscopicity, cost, and toxicity. In addition, cross sections embedded in $\mathrm{NaCl}$ were characterized by greater hardness, a feature that allowed us to obtain improved contact with the ATR crystal.
\end{abstract}

Published in the special issue Analytical Science in Italy with guest editor Aldo Roda.

S. Prati $\cdot$ G. Sciutto $\cdot$ E. Catelli $\cdot$ R. Mazzeo $(\triangle)$

Microchemistry and Microscopy Art Diagnostic Laboratory

(M2ADL), University of Bologna - Ravenna Campus,

via Guaccimanni 42,

48100 Ravenna, Italy

e-mail: rocco.mazzeo@unibo.it

A. Ashashina

JEOL Ltd,

1-2 Musashino 3-Chome Akishima,

196-8558 Tokyo, Japan
Keywords Preparation of cross sections · Fourier transform IR microscopy in attenuated total reflection mode $\cdot$ Paint samples $\cdot$ Spectral quality

\section{Introduction}

A polychrome sample is usually formed by overlaid paint layers of different thickness $(10-100 \mu \mathrm{m})$ applied over the support (canvas, wood, mortar) and composed of mixtures of organic and inorganic materials. In particular, a ground layer is often spread all over the support in order to reduce its porosity. One or several paint layers composed of a mixture of pigments or colorants and an organic binder may be applied directly on the preparatory layer or under a priming layer named "imprimatura" whose aim is to reduce further the porosity. A varnish is generally applied on the paint layers, aiming at protecting them and optimizing the chromatic effects.

The characterization and the stratigraphical location of the different paint components is of the outmost importance both for historical studies and for the identification of the most suitable conservation and restoration method to be adopted. The characterization of the painting technique, for instance, may support studies concerning authentication and stylistic attribution. In addition, stratigraphical analyses permit one to discriminate between original and overpainted layers, allowing restorers to decide which layers have to be removed during restoration.

The standard cross-section preparation is accomplished by embedding the paint fragment in a polymer resin in order to produce a block which allows one to easily handle small paint samples. Then the sample stratigraphy is obtained by grinding, polishing, or microtoming the block [1]. Penetration of the embedding resin (epoxy, polyester, acrylic, etc.) inside porous paint samples may negatively affect the 
characterization of the organic components, especially the uppermost varnish layers and those present in the preparatory ground. Moreover, smearing of the synthetic resin over the whole surface of the section may also occur during polishing. As a consequence, spectroscopic molecular investigations, such as Fourier transform IR (FTIR) microscopy analyses, may be strongly limited, owing to the overlapping of the absorption bands of the embedding resin and the organic paint components.

Several efforts have been devoted to developing different embedding procedures for the production of both thin sections and cross sections in the attempt to optimize the performances of different spectroscopic techniques and to prevent contamination by the embedding medium [1-18].

To this aim, IR-inactive salts, such as $\mathrm{AgCl}[10], \mathrm{KBr}[3,4$, $10,11,16], \mathrm{BaF}_{2}$, and $\mathrm{CaF}_{2}$ [11], were selected to produce thin sections and cross sections. Unfortunately, $\mathrm{AgCl}$ is photosensitive and darkens on exposure to light, making difficult the location of the sample within the block and its accurate microtoming to produce thin sections. $\mathrm{KBr}$ was investigated as an embedding medium for the production of thin sections, but it is too fragile for microtomy [10]. An alternative procedure for preparing thin sections embedded in $\mathrm{KBr}$ was proposed that involved polishing the $\mathrm{KBr}$ pellet on both sides [4]; however, the method did not allow homogeneous thickness to be obtained across the sample. Moreover, the operator's skill was particularly important to avoid the loss of sample, and cross sections prepared in this way were extremely fragile and difficult to handle. Conversely, more interesting results were obtained when $\mathrm{KBr}$ was employed for the preparation of cross sections $[3,11,16]$. Previous researches demonstrated that this sample preparation procedure is easy and allows one to prevent contamination by the embedding medium. Moreover, an improvement of the photogenic behavior of the cross section was also observed under UV light [3]. The use of a diamond microcompression cell was investigated as an alternative to microtomy for micro-FTIR ( $\mu$ FTIR) analyses of paint samples in transmission mode [17]. It is possible in this way to obtain a thin section, even if the compression caused deformation of the layers. Another published method used aluminum foil to create an effective barrier between the sample and the embedding resin. The sample is first wrapped in aluminum and then embedded and microtomed [6]. However, this approach requires relatively large samples and it may be difficult to employ when real small samples have to be handled.

To overcome the infiltration of the embedding resin in porous samples, de Fonjaudran et al. [9] proposed a method based on the use of cyclododecane as a temporary barrier. This compound is capable of subliming at room temperature and can be used to encapsulate the sample before embedding it in an acrylic resin. The block is then microtomed to obtain a cross section so that smearing of the resin is avoided. However, upon sublimation of cyclododecane, a gap was produced around the sample, thus reducing its stability.

Recently, our research group focused on the evaluation of the performance of attenuated total reflection (ATR) FTIR microscopy when applied to paint cross sections prepared with different procedures [16].

ATR FTIR microscopy was chosen because it has been widely employed for the analysis of both unmounted fragments and samples prepared as cross sections thanks to its capability of characterizing both organic and inorganic compounds that are active in the mid-IR spectral region. Our research allowed the selection of three preparation procedures as the most promising: (1) the use of $\mathrm{KBr}$ as an embedding system, (2) pretreatment with cyclododecane, and (3) the use of argon ion milling to polish the surface of samples embedded in synthetic resin [16]. One limitation of the $\mathrm{KBr}$ embedding procedure was that the operator's skill was crucial to obtain flat sections without damaging the samples. Moreover, dry polishing may produce other sources of contamination which may affect the analytical results. In particular, the presence of hygroscopic $\mathrm{KBr}$ particles on the sample may lead to noisy IR spectra in the $1,600-1,800-\mathrm{cm}^{-1}$ spectral range and to consequent reduction of the spectral quality.

In the cyclododecane-treated sample, the contamination was reduced, even though the characterization of external layers may be compromised. In addition, the morphology of the surface may negatively influence the analytical results because contact between the ATR crystal and the sample may not be good in the most irregular areas. Indeed, it is known that good contact between the sample and the ATR crystal is crucial to obtain well-resolved and intense spectra. Samples embedded in a synthetic resin and polished by means of argon ion milling provided the best results when both specular reflection [13] and ATR [16] measurements were performed. However, one drawback of the method was that, apart from being expensive and time-consuming, the external layers may be partially or completely covered by the embedding resin [16].

Within this scenario, the present research focused on the development of innovative embedding procedures able to overcome the drawbacks of the $\mathrm{KBr}$ method. The first procedure foresaw the use of $\mathrm{KBr}$ as a barrier against the contamination by the embedding synthetic medium. To this aim the sample was firstly embedded in $\mathrm{KBr}$ and then the pellet was embedded in resin, in order to obtain a block that could be polished manually using a sample holder. As already reported [18], the use of a sample holder allows one to improve the flatness of cross sections embedded in synthetic resins. Thus, the operator's skill was less crucial because the system itself allows a more controlled and reproducible pressure to be applied on the sample.

In addition, to obtain regular surfaces, argon ion milling was tested as a procedure for polishing samples embedded 
in $\mathrm{KBr}$. In this way, an increase in spectral quality was obtained, which was also connected to a reduction of the contamination effects produced by dry polishing. However, this method is expensive and time-consuming and can be difficult to apply in routine analyses.

Finally, the use of $\mathrm{NaCl}$ as an alternative to $\mathrm{KBr}$ was proposed. This salt has some advantages in terms of hygroscopicity (the solubility of $\mathrm{NaCl}$ is 35.8 g per $100 \mathrm{~g}$ of $\mathrm{H}_{2} \mathrm{O}$, whereas the solubility of $\mathrm{KBr}$ is $67.8 \mathrm{~g}$ is $100 \mathrm{~g}$ of $\mathrm{H}_{2} \mathrm{O}$ ), cost, and toxicity. Generally, $\mathrm{NaCl}$ is not used for the preparation of pellets because its IR-accessible spectral range is between 4,000 and $650 \mathrm{~cm}^{-1}$, whereas the transparent region of $\mathrm{KBr}$ is wider $\left(4,000-400 \mathrm{~cm}^{-1}\right)$. Although this aspect is particularly relevant when FTIR analyses are performed with traditional spectrophotometers, it becomes negligible when FTIR microscopy is used. Indeed mercury cadmium telluride and focal plane array detectors already produce a cutoff in the investigated spectral region down to $670 \mathrm{~cm}^{-1}$ and $900 \mathrm{~cm}^{-1}$, respectively.

The three proposed procedures were used for the preparation of both reconstructed and real paint cross sections. The surface morphology produced by all methods was evaluated by means of confocal microscopy and the degree of contamination by the embedding medium $(\mathrm{KBr}, \mathrm{NaCl}$, and synthetic resin) was also considered. Moreover, the abovementioned issues were taken into consideration to evaluate the effect of sample preparation on the results achievable by means of $\mu$ FTIR microscopy in ATR mode.

\section{Materials and methods}

\section{Materials}

Inorganic salts $(\mathrm{KBr}$ and $\mathrm{NaCl})$ of analytical grade purchased from Sigma-Aldrich (St. Louis, MO, USA) was used. Polyester resin (SeriFix Resin and SeriFix Hardener) for embedding samples were purchased from Struers (Ballerup, Denmark).

Standard samples were collected from paint reconstructions prepared in the laboratory according to ancient treatises [19] and simulating the stratigraphy of ancient easel paintings. Pigment layers, applied over a preparatory layer of gypsum and rabbit glue, were obtained using a mixture of lead white with linseed oil (LW), smalt, and malachite with whole egg binder. Pigments and gypsum were purchased from Zecchi (Florence, Italy), and rabbit glue and linseed oil were from Phase (Bologna, Italy).

The $\mathrm{NaCl}$ embedding procedure was applied on paint reconstructions containing malachite in order to investigate the effect of chloride on copper-based pigments.

$\mathrm{The} \mathrm{KBr} /$ synthetic resin embedding (KRE) system was tested on a real sample from the Greek icon Last Judgment painted in 1771 by Ioannis [20]. A similar fragment from the same icon was embedded with the traditional $\mathrm{KBr}$ method for comparison.

The KBr embedding procedure followed by polishing with argon ion milling was tested on a sample from a wood panel painting attributed to Baldassarre Carrari (1460-1516) [16].

\section{$\mathrm{KBr} /$ synthetic resin embedding (KRE) system}

Samples were previously embedded in $\mathrm{KBr}$ following a standard procedure [3]. Briefly, the microfragment was placed in a macro-micro pellet die where a previous $\mathrm{KBr}$ pellet-bed ( $2 \mathrm{t}$ for $1 \mathrm{~min}$ ) had been prepared, covered with additional $\mathrm{KBr}$, and then pressed ( $3 \mathrm{t}$ for $2 \mathrm{~min}$ ). Afterwards, the pellet was reduced in size and submitted to the polyester resin embedding procedure (Fig. 1a). Dry polishing was performed using silica abrasive papers (purchased from Micro-Surface Finishing Products, Wilton, IA, USA) in grades from 1,000 to 12,000 , to obtain a high-quality surface in terms of planarity and roughness. A polishing sample holder was employed to ensure a high level of surface planarity (Fig. 1b).

To evaluate the performance of the proposed approach, two samples collected from the same paint reconstruction were embedded following the KRE procedure and polished with and without the use of the sample holder. To better compare the two approaches, the duration of each finishing polishing step was fixed as reported in Table 1.

$\mathrm{KBr}$-embedded samples polished with argon ion milling (KAIM)

KBr-embedded samples polished with argon ion milling (KAIM) were obtained by embedding the sample in $\mathrm{KBr}$ following a standard procedure [3]. Then the cross sections, after being dry-polished using silicon carbide papers up to grade 12,000 , were subjected to ion milling employing a JEOL IB-09010 cross-section polisher with ultrapure argon (99.9999\%, purity grade 6.0).

The cross-section polisher uses a beam of accelerated argon ions to polish the material of interest. By placement of a shield that is resistant to the argon ion beam on the top of the sample, a portion of the sample is protected from the abrasive argon ion beam. Because the rate of attrition is effectively zero when the surface of the material is parallel to the argon ion beam, a very flat cross section is formed. It is free from contamination and damage associated with other types of polishing, such as mechanical or chemical etching.

The polishing was performed at $5 \mathrm{kV}$ for $10-15 \mathrm{~h}$.

\section{Embedding in $\mathrm{NaCl}$}

Paint fragments were placed on a soft base, which was obtained by pressing $300 \mathrm{mg}$ of $\mathrm{NaCl}$ into a pellet under 
Fig. 1 a The $\mathrm{KBr} /$ synthetic resin embedding procedure. b The polishing sample holder a

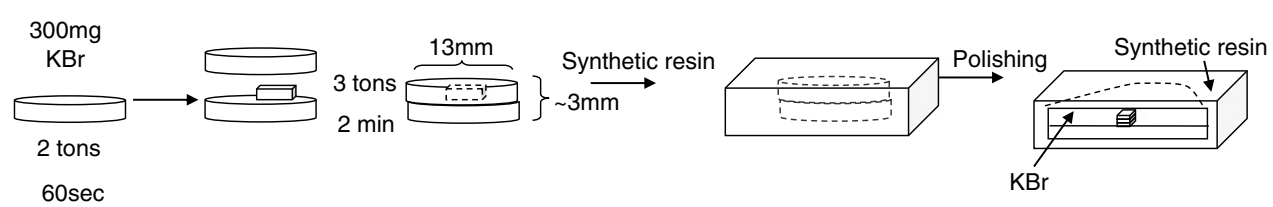

b

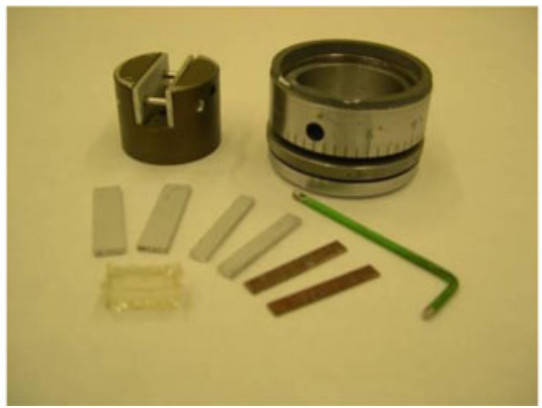

low pressure ( $1 \mathrm{t}$ for $10 \mathrm{~s}$ ). Then, $300 \mathrm{mg}$ of $\mathrm{NaCl}$ was added and a pressure of $3 \mathrm{t}$ was applied for $2 \mathrm{~min}$. Afterwards, the pellet (13-mm diameter and $2 \mathrm{~mm}$ thick) was carefully extracted from the holder. Finally, the cross section was obtained by dry polishing on silicon carbide papers (Micro-Mesh $^{\circledR}$ ) from grade 1,000 to grade 12,000 .

For the preparation of the $\mathrm{NaCl} /$ synthetic resin embedded samples, the procedure described for $\mathrm{KBr}$ was followed.

\section{Instrumentation}

Dark-field observations were performed using an Olympus BX51M optical microscope equipped with fixed $\times 10$ oculars and objectives with different magnifications $(\times 5, \times 10$, $\times 20, \times 50$, and $\times 100$ ). Visible radiation and UV radiation were provided by a $100-\mathrm{W}$ halogen projection lamp and an Ushio Electric USH102D lamp, respectively. Cross-section photomicrographs were recorded with an Olympus DP70 digital scanner camera directly connected to the microscope.

The recording of the morphology of the sample surface was performed with the use of a LEXT microscope equipped with a 408-nm laser diode purchased from Olympus, combined with optics specifically designed for operation at this wavelength. Two-dimensional and three-dimensional images were acquired at different magnifications $(\times 5, \times 10, \times 20$, and $\times 50)$.

Table 1 Polishing steps followed in the preparation procedure with and without the sample holder

\begin{tabular}{ll}
\hline SiC paper grade & Time $(\mathrm{min})$ \\
\hline 1,000 & 1 \\
6,000 & 2 \\
8,000 & 4 \\
12,000 & 8 \\
\hline
\end{tabular}

Roughness profiles were obtained and area measurements were performed.

The elemental composition was determined using a Zeiss EVO 50 EP extended-pressure scanning electron microscope (SEM) equipped with an INCA energy-dispersive $\mathrm{X}$-ray (EDX) detector. Data analysis was performed with the use of INCAEnergy.

Moreover, a SEM instrument (JSM-6610) developed by JEOL, coupled with a low-vacuum chamber, was also used for elemental and morphological analysis of samples before and after ion polishing.

The FTIR mapping measurements were performed in ATR mode using a Thermo Nicolet iN ${ }^{\text {TM }} 10 \mathrm{MX}$ raster scanning microscope, fitted with a mercury cadmium telluride detector cooled by liquid nitrogen and a conical germanium crystal. Spectra were recorded in the range 4,000-675 $\mathrm{cm}^{-1}$. Data collection and postrun processing were performed using OMNIC Picta ${ }^{\mathrm{TM}}$ (Thermo).

\section{Results and discussion}

Development of improved $\mathrm{KBr}$ embedding methods

It is known that the $\mathrm{KBr}$ embedding method has some specific drawbacks mainly related to the brittleness and hygroscopicity of $\mathrm{KBr}$, which may affect the physical stability of cross sections [16]. Moreover, the operator's skill is of the outmost importance in order to obtain uniform and flat surfaces, which are fundamental to guarantee efficient contact between the ATR crystal and the sample and to obtain well-resolved spectra and reproducible results. In the attempt to overcome these disadvantages, two strategies were developed. 


\section{$\mathrm{KBr} /$ synthetic resin embedding (KRE) system}

The KRE system foresaw the use of an external coating made of a polymeric resin aimed at the physical stabilization of the $\mathrm{KBr}$ pellets as well as at protecting the salt from atmospheric humidity. On the other hand, the presence of the salt can be considered as a barrier against the infiltration of the resin into the porous layers of the paint sample. A significant advantage was achieved through the use of a polishing sample holder coupled with suitable supports designed to fit with paint cross sections embedded in resin (Fig. 1). As previously observed on samples embedded in organic resins [18], the holder was used to reduce the influence of the operator's skill during the preparation procedures, thus obtaining more constant and reproducible results.

Images acquired with the laser confocal microscope (Fig. 2) allowed us to compare manual and holdercontrolled polishing procedures applied on LW paint reconstructions (for the composition, see "Materials") embedded

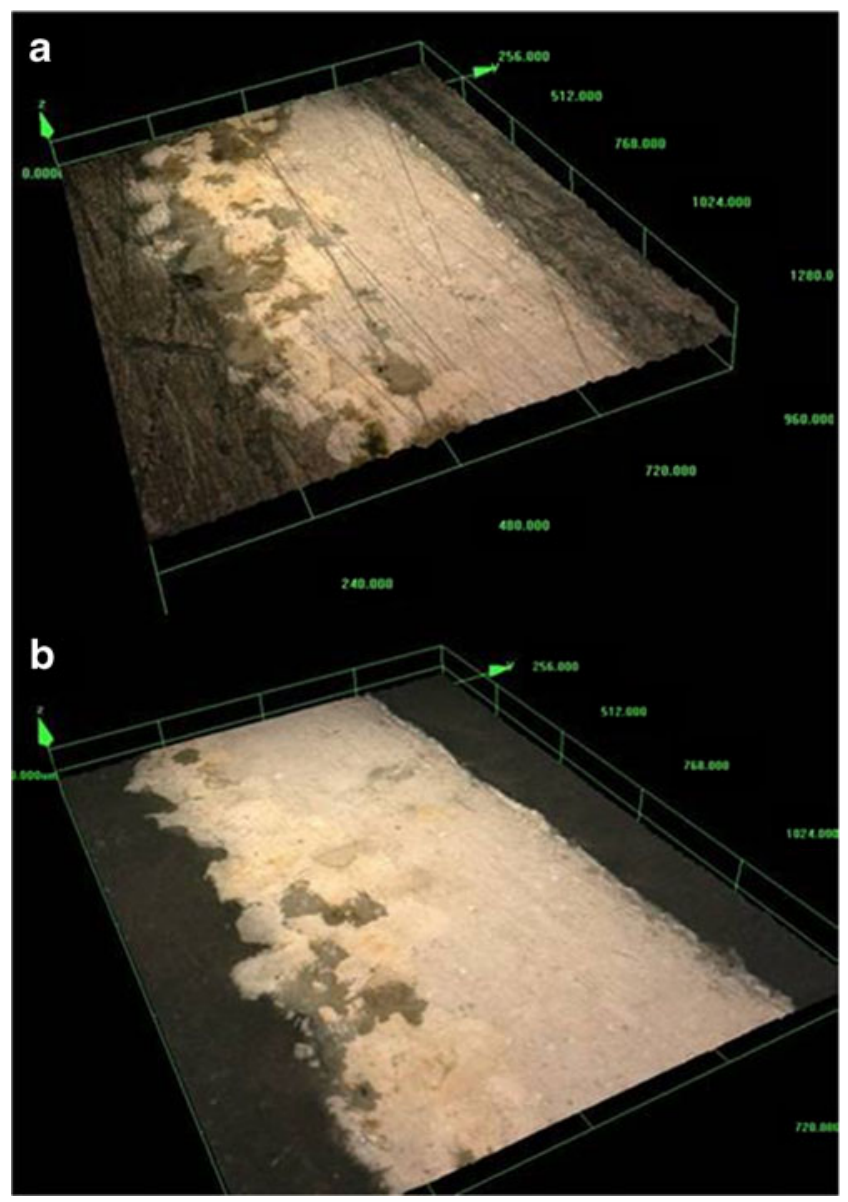

Fig. 2 Three-dimensional color confocal images (magnification $\times 200$ ) of a cross section of a mixture of lead white with linseed oil (LW) embedded with the $\mathrm{KBr} /$ synthetic resin embedding (KRE) procedure and polished $\mathbf{a}$ without and $\mathbf{b}$ with the use of the polishing sample holder with the KRE procedure. As expected, the use of an appropriate sample holder was particularly helpful because the constant and homogeneous pressure on the whole crosssectioned surface allowed a high-quality surface in terms of roughness to be obtained.

Specific investigations were conducted to identify the presence of polyester embedding resin on the $\mathrm{KBr}$ barrier and on the sample layers. Luckily, the results obtained by $\mu$ FTIR analyses in ATR mode on standard cross sections showed that the contamination affected just a few micrometers of the salt, directly in contact with the resin, thus allowing the characterization and location of both organic and inorganic substances. As shown in Fig. 3, the white paint layer was well recognizable in the chemical map by mapping the absorption band at $1,735 \mathrm{~cm}^{-1}$ for the identification of oil and the marker band at $838 \mathrm{~cm}^{-1}$ related to the presence of lead white. Moreover, gypsum was localized within the preparatory layer by mapping the band at about $1,100 \mathrm{~cm}^{-1}$.

As a drawback, SEM-EDX analyses showed that $\mathrm{KBr}$ is spread on the sample surface in both manually and holdercontrolled polished cross sections (data not shown).

The proposed method was tested and applied on a real sample collected from a Greek icon from the eighteenth century. The results obtained by $\mu$ FTIR mapping analyses in ATR mode were compared with those obtained on the sample embedded with the traditional $\mathrm{KBr}$ procedure (see the photomicrographs of the two cross sections in Fig. 4). It is worth highlighting that the present research was aimed not at identifying the exact composition of the real sample, but at characterizing whether the sample preparation procedures may affect the analytical performance of investigations by means of $\mu$ FTIR analysis in ATR mode.

The analyses performed on the two samples allowed us to detect the same components. The brownish-yellow ground was composed of gypsum and a proteinaceous material (layer 0), over which a blue layer (layer 1) characterized by the presence of irregular dark-blue particles $(5-20 \mu \mathrm{m})$ of Prussian blue $\left(\mathrm{Fe}_{4}\left[\mathrm{Fe}(\mathrm{CN})_{6}\right]_{3} \cdot n \mathrm{H}_{2} \mathrm{O}\right)$, mixed with lead white, $2 \mathrm{PbCO}_{3} \cdot \mathrm{Pb}(\mathrm{OH})_{2}$, and a proteinaceous binder were applied. The spectra recorded from the external white fluorescent layer (layer 2) were noisy but suggested the presence of a lipidic material, calcium oxalate, and lead carboxylates, probably formed by the interaction between the lipidic binder and the above-mentioned lead white.

As observed in the paint reconstructions embedded with the KRE system, also in the icon sample the synthetic resin was present just on $\mathrm{KBr}$ in the first few micrometers near the edge between the resin and the salt and did not contaminate the sample.

Moreover, the KRE method produced positive results in term of spectral quality. In particular, the spectra recorded from the preparatory ground layer of the sample embedded 
Fig. 3 Cross-section microphotograph of the LW sample under visible light $(a)$ and Fourier transform IR (FTIR) false-color plots representing the following peak area profiles: $852-820 \mathrm{~cm}^{-1}$ (b; lead white), $1,754-1,712 \mathrm{~cm}^{-1}$ ( $c$; siccative oil), and 1,170 $1,016 \mathrm{~cm}^{-1}$ ( $d$; gypsum)
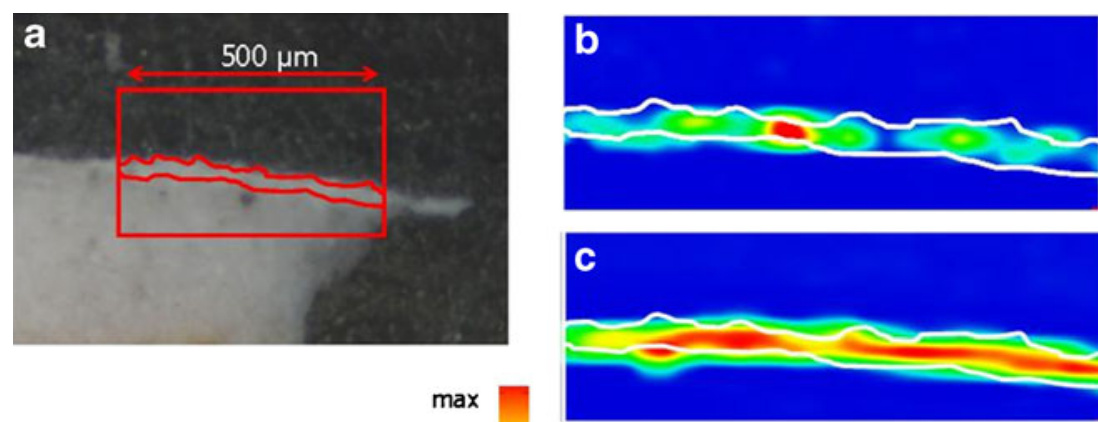

$\max$

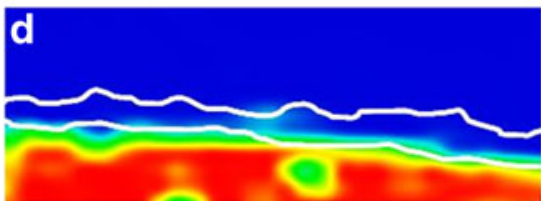

with the KRE procedure showed, at about 3,400 and $3,520 \mathrm{~cm}^{-1}$, more defined and less noisy $\mathrm{OH}$ bands typical of gypsum, as well as amide I and amide II bands (Fig. 5).

In addition, the use of the sample holder had the advantage of reducing the influence of the operator's skill. Indeed, the polishing procedure can be easily standardized by fixing the duration of each finishing step and because the applied pressure depends mainly on the sample holder (Table 1).

\section{KBr-embedded samples polished with argon ion milling (KAIM)}

The effectiveness of ion milling polishing procedure has already been proved [13, 16]. However, this method has never been tested on $\mathrm{KBr}$-embedded cross sections. In particular, this approach could be considered extremely powerful in reducing contamination of the sample by paint layers and embedding material $(\mathrm{KBr})$ and in obtaining a higher surface quality. Firstly, to verify the stability of the embedding medium under ion bombardment, the polishing procedure was applied on paint reconstructions embedded in $\mathrm{KBr}$ with the traditional method and polished in dry conditions up to grade 12,000. The confocal images of the BO sample prepared with the KAIM method showed a more planar and regular surface (Fig. 6a) when compared with the sample embedded with the KRE procedure and polished either manually or with the support of the sample holder (Fig. 2). Even if a crack was present at the edge between the painted layer and $\mathrm{KBr}$, the backscattering images of the sample collected in topographic mode before and after $10 \mathrm{~h}$ treatment at $50 \mathrm{kV}$ showed that the crack was already present in the sample before the argon ion milling (Fig. 6b, c). Moreover, it may be noticed that the morphology of the sample was completely different before the ion bombardment. In
Fig. 4 Photomicrographs of the blue icon samples. Cross section obtained with the traditional $\mathrm{KBr}$ embedding procedure: a visible microscopic image; b fluorescent image after UV illumination. Cross section obtained with the KRE embedding procedure: c visible microscopic image; $\mathbf{d}$ fluorescent image after UV illumination
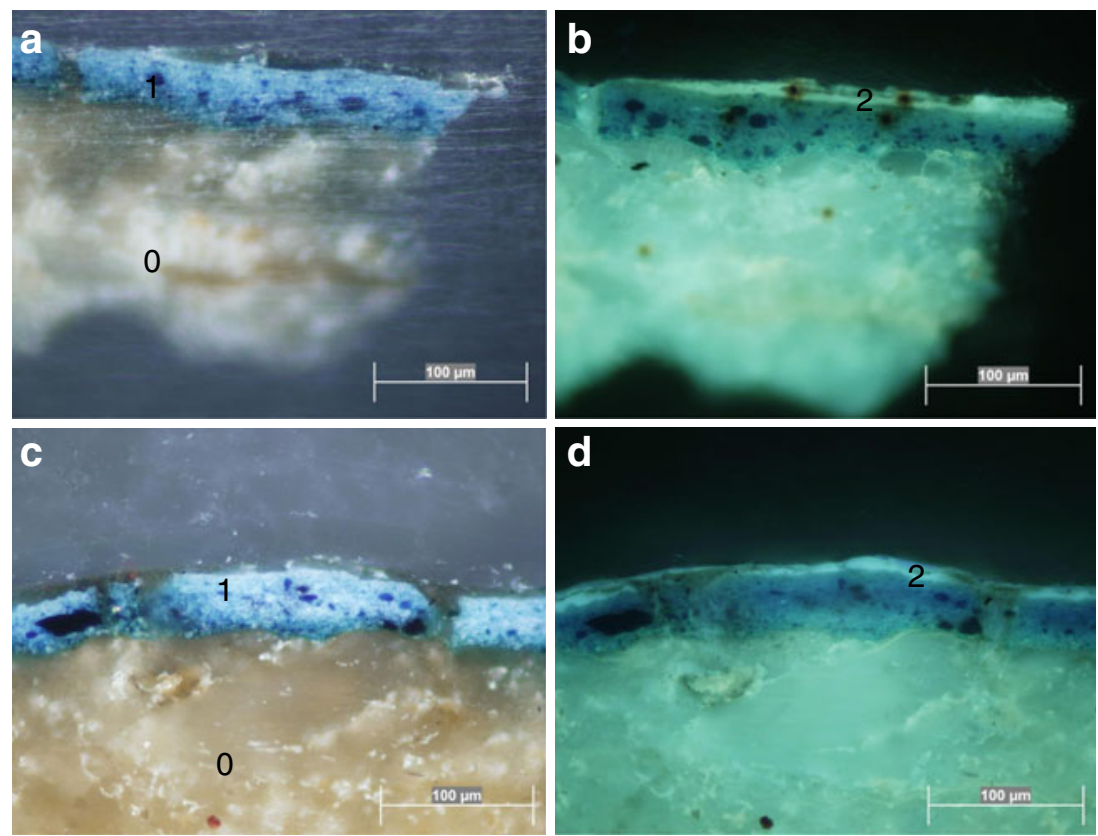
Fig. 5 Spectra recorded by FTIR microscopy in attenuated total reflection (ATR) mode on the preparatory layer of the cross sections of the icon: $a$ embedded with the traditional $\mathrm{KBr}$ method and $b$ embedded with the KRE method

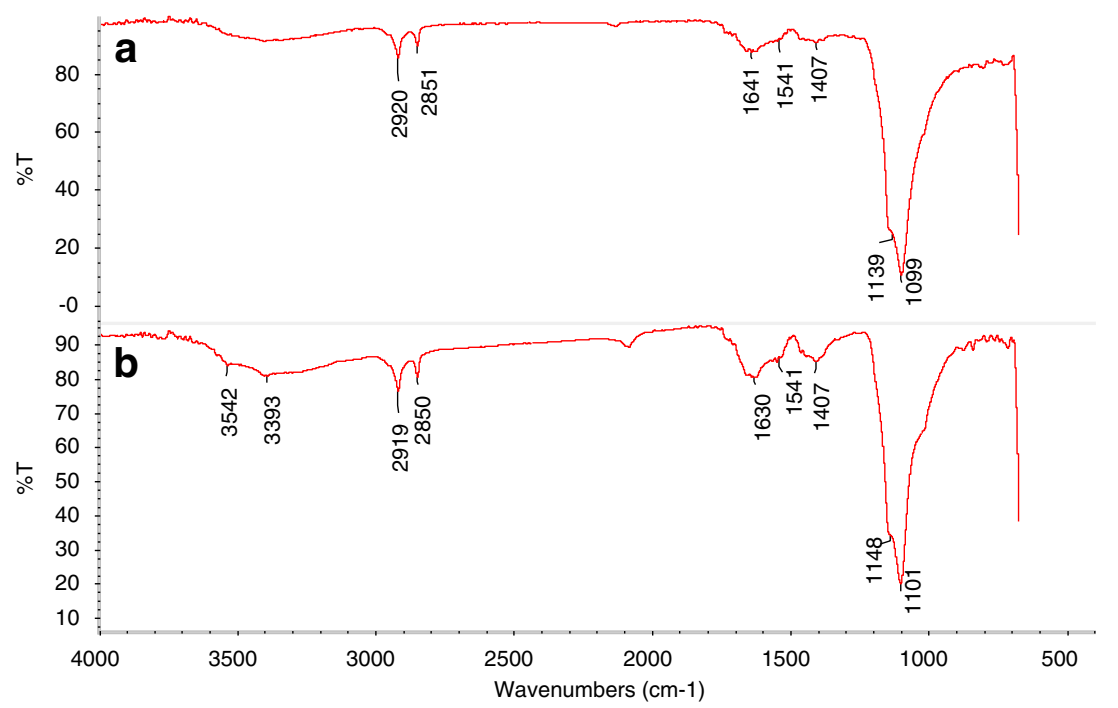

particular, the EDX elemental map showed that before the ion polishing the inner layer was completely covered by $\mathrm{KBr}$. This demonstrated how the traditional $\mathrm{KBr}$ embedding system may not guarantee reproducibility of results, which strongly depends on the operator's skills. Moreover, dry polishing can lead to contamination effects if the polishing papers are not accurately cleaned and not often replaced during preparation of the cross section. Elemental analyses were performed after ion milling in order to investigate if the typical contamination by $\mathrm{KBr}$ particles caused by the preliminary dry polishing may be eliminated. By comparing elemental maps of $\mathrm{K}$ and $\mathrm{Br}$ before and after the treatment, we verified that their intensity seemed to decrease all over the sample surface. However, single-point analyses showed that $\mathrm{KBr}$ was still partially present on the different layers.

To better understand the effect of this sample preparation on real samples, the KAIM method was applied on a fragment collected from the Virgin's halo of a panel painting attributed to Baldassarre Carrari (ROND13). Fragments from the same area were the subject of a recent article aimed
Fig. 6 a Three-dimensional color confocal images (magnification $\times 200$ ) of the LW cross section embedded in $\mathrm{KBr}$ and polished by argon ion milling, and backscattering images in topographic mode of the LW cross section $\mathbf{b}$ before argon ion milling and $\mathbf{c}$ after $10 \mathrm{~h}$ argon ion milling at $50 \mathrm{kV}$
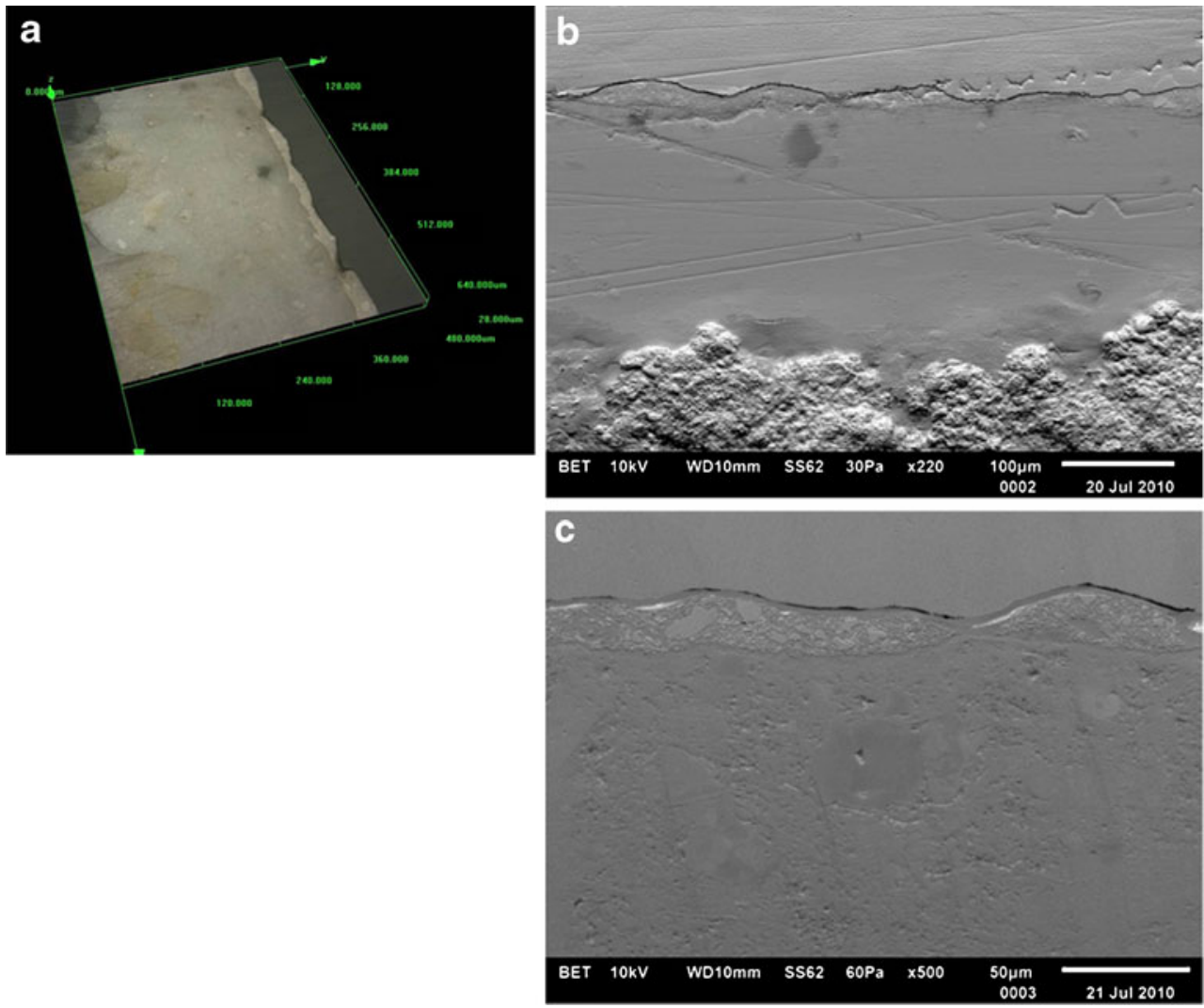
at comparing the performance of FTIR microscopy in ATR mode on the same sample embedded with seven different preparation procedures [16]. In particular, a sample embedded in $\mathrm{KBr}$ with the traditional method and a sample embedded in polyester resin and polished with argon ion milling were studied in the work mentioned and the related results are considered as a reference in the following discussion.

According to the previous diagnostic campaign, analyses performed on the sample prepared with the KAIM method allowed the characterization and location of different organic and inorganic compounds within the sample (the photomicrograph obtained under visible light with indication of the different layers is reported in Fig. 7d). The preparatory layer was composed of gypsum (layer 0 ), probably with a glue binder, over which a layer of lead white mixed with a lipidic binder was applied (layer 1). Three green layers (layers 2-4) above this layer were composed of copper acetate (verdigris) or resinate in an oily binder. In layer 3 , white particles ascribable to lead white were identified. All the spectra related to layers 2-4 were dominated by bands in the ranges $1,547-1,555 \mathrm{~cm}^{-1}$ and $1,404-1,410 \mathrm{~cm}^{-1}$. Such bands may be related to some copper complexes or to lead acetate, as degradation products, which may possibly be formed by the interaction of the original verdigris and lead white. A layer of a clay-type earth pigment and oil was spread on the green background (layer 5) to bind the gold leaf of the Virgin's halo. A final varnish was present as a protective layer (layer 6) [16].

Confocal microscope analyses of the three samples after polishing were comparable even though the two cross sections polished with argon ion milling seemed to be more planar (Fig. 7a) [16]. However, ion milling enlarged some cracks present on the cross section before the treatment as evidenced by SEM-EDX images (Fig. 7b, c).
Optical microphotographs under UV light showed that the external protective layer was not visible in the sample embedded in resin and then polished with argon ion milling, and it was identified only in the cross sections embedded in $\mathrm{KBr}$ [16]. As expected, the varnish layer was also visible in the sample prepared with the KAIM method, as reported in Fig. 7e. Concerning the contamination effects, SEM-EDX analyses of the $\mathrm{KBr}$-embedded samples showed that $\mathrm{KBr}$ particles were present both in manually polished samples and in samples polished by argon ion milling, but in the latter samples the EDX peaks were less intense. Moreover, a previous study showed that IR spectra recorded on a sample embedded in $\mathrm{KBr}$ and polished manually were particular noisy in the region between 1,800 and $1,600 \mathrm{~cm}^{-1}$, probably owing to the presence of hygroscopic $\mathrm{KBr}$ particles [16]. Conversely, spectra of the sample prepared with the KAIM method were characterized by a higher spectral quality in terms of signal-to-noise ratio. The reason is that better contact was achieved and the sample was less contaminated by the embedding medium. As an example, the $\mathrm{OH}$ bands of the clay (layer 5) were clearly visible only in the sample polished with ion milling (Fig. 8, spectra a and b). Moreover, in the sample prepared with the KAIM method, the band associated with verdigris in layers 2-4 at about $1,600 \mathrm{~cm}^{-1}$ appeared more evident, whereas in the manually polished sample, only a shoulder was identified (Fig. 8, spectra c and d).

\section{Development of the $\mathrm{NaCl}$ embedding method}

$\mathrm{NaCl}$ was tested as an embedding medium as an alternative to $\mathrm{KBr}$ because it has several advantages in terms of hygroscopicity, cost, and toxicity.

Paint reconstructions were embedded both with the single embedding procedure and with the $\mathrm{NaCl} /$ synthetic resin
Fig. 7 a Three-dimensional color confocal images (magnification $\times 200$ ) of sample ROND13 embedded in $\mathrm{KBr}$ and polished by argon ion milling, backscattering images of sample ROND13 b before argon ion milling and $\mathbf{c}$ after $10 \mathrm{~h}$ argon ion milling at $50 \mathrm{kV}$, and cross-section photomicrographs of sample ROND13 after argon ion milling obtained under $\mathbf{d}$ visible and $\mathbf{e} \mathrm{UV}$ illumination
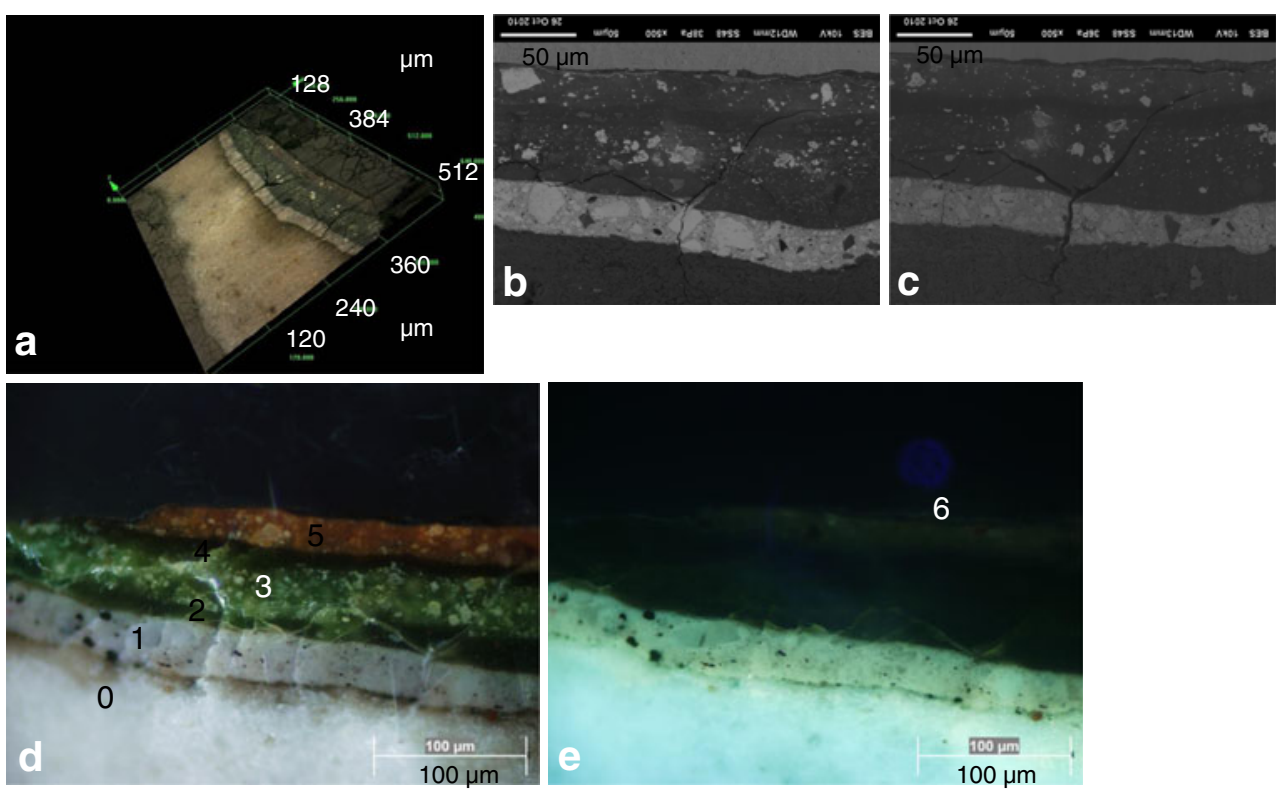
Fig. 8 Spectra obtained on sample ROND13 with microFTIR in ATR mode on layer 5 (earth-type pigment): $a$ sample polished by argon ion milling and $b$ sample polished manually. Spectra obtained on sample ROND13 with micro-FTIR in ATR mode on layer 3: $c$ sample polished by argon ion milling and $d$ sample polished manually
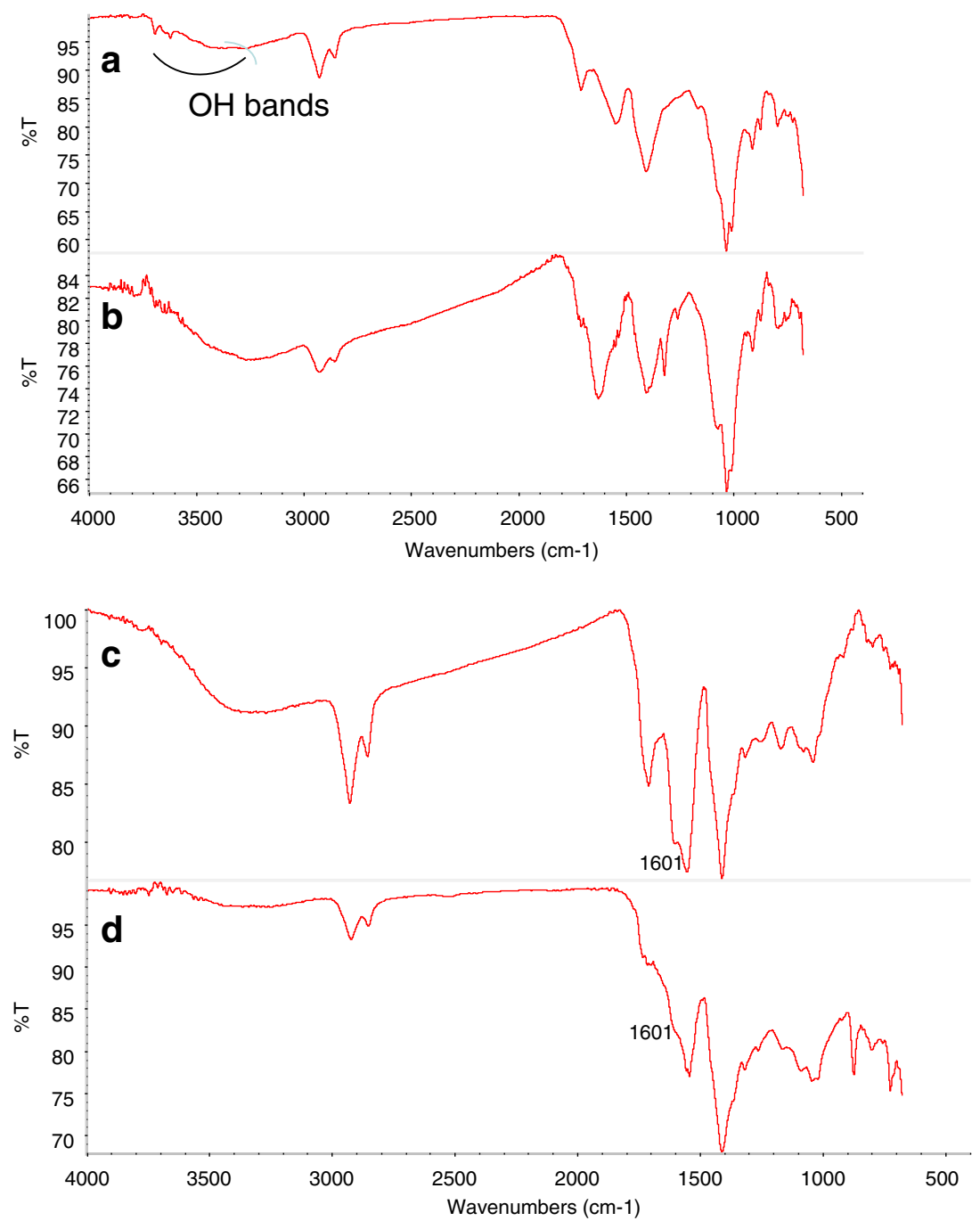

embedding procedure as reported in "Embedding in $\mathrm{NaCl}$. " Observations by confocal microscopy allowed us to confirm that, as described for $\mathrm{KBr}$, both methods permitted us to obtain good surface planarity, but the use of the sample holder made the procedure less dependent on the operator's skill.

Particular attention was paid to the evaluation of the performance of the new embedding salt in terms of physical stability. Confocal microscopy observations of both $\mathrm{NaCl}-$ embedded and $\mathrm{KBr}$-embedded LW samples were performed after ATR mapping analyses. The three-dimensional confocal images showed that $\mathrm{NaCl}$-embedded samples had greater physical resistance to the ATR crystal pressure than $\mathrm{KBr}-$ embedded samples (Fig. 9). Consequently, a lower pressure was sufficient to obtain good contact, thus improving the quality of the spectrum and reducing the slipping of the samples after contact with the ATR crystal [16].

The $\mathrm{NaCl}$ contamination on the paint layers was evaluated through EDX elemental maps, which revealed the presence of a few grains of the salt in the cavities of the cross section (data not shown). To reduce this effect, the use of argon ion milling is proposed.
Smalt cross sections (for the composition, see "Materials") were prepared using the $\mathrm{KRE}$ and $\mathrm{NaCl} /$ synthetic resin embedding procedures in order to evaluate the performance of FTIR microscopy in ATR mode. Three different cross sections were prepared for each method (in total six) and for each of them three replica spectra were recorded at different pressures (3\%, $5 \%, 8 \%, 10 \%, 20 \%)$ on the painted layers. Thus, a total of nine replicas for each pressure and for each preparation method were obtained. In Fig. 10 the ratio between the amide I and amide II bands and the ratio between the $1,000-\mathrm{cm}^{-1}$ and $767-\mathrm{cm}^{-1}$ bands related to $\mathrm{Si}-\mathrm{O}-\mathrm{Si}$ vibrations are reported to evaluate the reproducibility of the acquired spectra.

The ratios related to the samples embedded in $\mathrm{KBr}$ were characterized by a larger variability with respect to those obtained for the samples embedded in $\mathrm{NaCl}$. Moreover, even though the intensity of the signal of each component is connected to its local concentration, which was not homogeneous, on average, signals recorded from $\mathrm{NaCl}-$ embedded samples were more intense and less noisy at each pressure. Conversely, the signal of the proteinaceous binder in the $\mathrm{KBr}$ samples was generally less intense and the 
Fig. 9 Three-dimensional confocal images of the LW sample a embedded in $\mathrm{NaCl}$ after ATR analyses and $\mathbf{b}$ embedded in $\mathrm{KBr}$ after ATR analyses
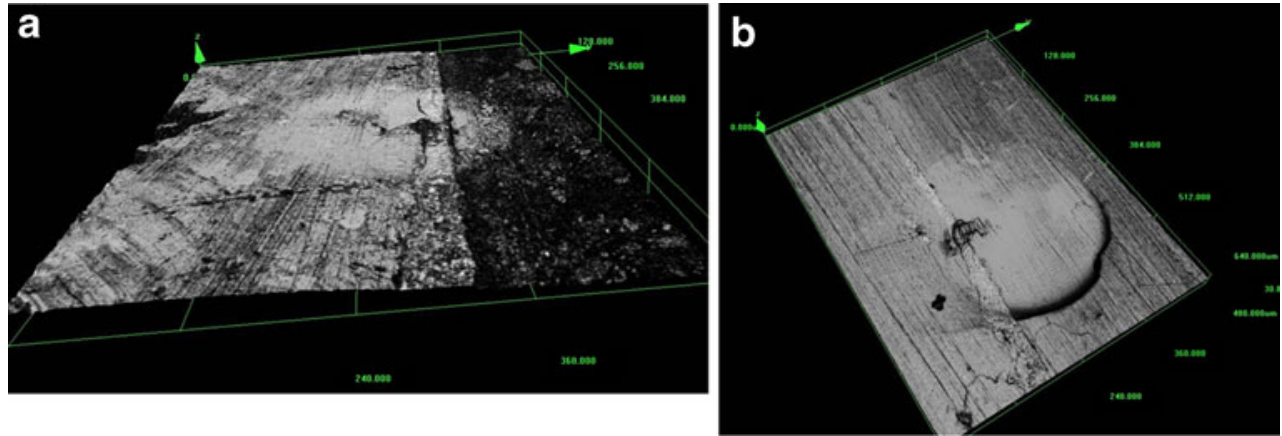

highest signals were obtained only if a pressure up to $20 \%$ was applied.

It was interesting to note that this finding may be connected to the greater hardness of $\mathrm{NaCl}$-embedded cross sections compared with the $\mathrm{KBr}$-embedded ones, which may improve the reproducibility and stability of the contact between the sample surface and the ATR crystal. Another aspect which can affect the spectral quality may be ascribed to the different hygroscopicity of the embedding materials. As shown in a recently published article [16], $\mathrm{KBr}$ particles on the surface of the cross sections lead to noisy spectra in the region between 1,600 and $1,800 \mathrm{~cm}^{-1}$. Conversely, $\mathrm{NaCl}$ is less hygroscopic, and

\section{a}

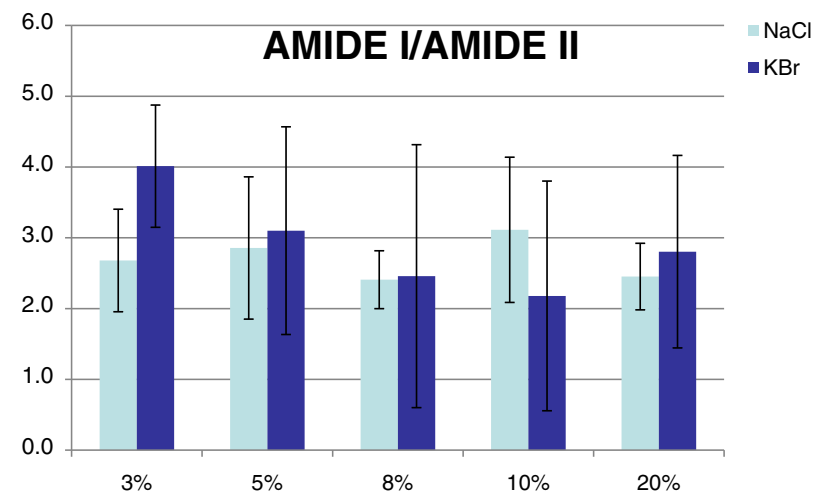

b

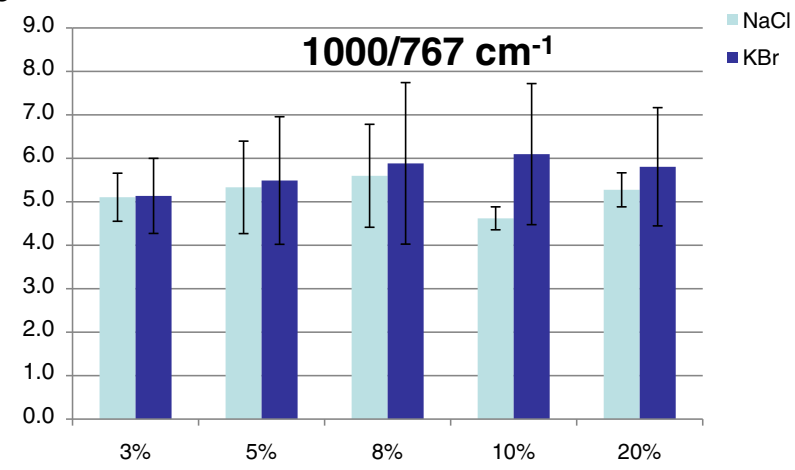

Fig. 10 a Amide I/amide II ratio and b $1,000 \mathrm{~cm}^{-1} / 767 \mathrm{~cm}^{-1}$ ratio obtained in spectra recorded from the painted layer of smalt paint reconstructions embedded with both the KRE procedure and the $\mathrm{NaCl} /$ synthetic resin embedding procedure its presence on the surface of the cross section probably had less effect on the spectral quality.

Standard paint reconstructions containing malachite were analyzed in order to evaluate if $\mathrm{NaCl}$ may affect copperbased pigments, promoting the formation of copper chloride salts. The cross sections prepared with the $\mathrm{NaCl} /$ synthetic resin embedding procedure were stored either in a heated IR cabinet or in a climate chamber containing a beaker with $100 \mathrm{~mL}$ of water and analyzed. No trace of chlorides was detected over the cross sections after periods of 4 and 8 months, neither on the ones kept in the cabinet nor on the ones kept under high-humidity conditions. However, this finding is currently under investigation to evaluate the effect of a longer exposure period.

\section{Conclusion}

The present research allowed the successful development and evaluation of three new methods that make use of IRinactive salts for the preparation of paint cross sections.

The well-known $\mathrm{KBr}$ embedding method was optimized using the inorganic salt as a barrier against infiltration of the resin. The KRE system showed better performance in terms of physical stability, and $\mu$ FTIR analyses allowed us to exclude the presence of contamination caused by the smearing of the resin during the polishing procedure. In addition, the method allowed us to obtain less fragile samples suitable to be polished by means of a sample holder. Thus, the quality of the cross sections obtained was less affected by the operator's skill, and improvement of the spectral quality was observed in $\mu$ FTIR analyses performed in ATR mode.

Argon ion milling applied on $\mathrm{KBr}$-embedded samples provided very promising results in terms of sample planarity and reduction of superficial contamination by KBr. These improvements also led to an increase in the spectral quality achievable with $\mu$ FTIR analyses in ATR mode. With respect to the samples embedded in resin and submitted to the ion bombardment, comparable results may be obtained concerning sample planarity and ATR spectral quality. However, the outermost layers (e.g., varnishes) are often contaminated by the embedding resin and can hardly be 
analyzed in these samples; on the other hand, the $\mathrm{KBr}$ embedding procedure still allows the external substances to be detected and identified.

The argon ion milling polishing procedure is extremely time-consuming and expensive, so it cannot be used in routine analyses and can only be used for important and rare paint samples.

The third proposed procedure, which involves the use of $\mathrm{NaCl}$, allowed us to obtain harder cross sections, thus contributing to improvement of the contact with the ATR crystal and requiring the application of less pressure than for the $\mathrm{KBr}$ embedded samples. Moreover, compared with $\mathrm{KBr}, \mathrm{NaCl}$ has some advantages in terms of hygroscopicity, cost, and toxicity. However, chloride ions could interact with copper-based substances to form copper chloride. Preliminary tests performed on cross sections containing malachite and submitted to both high-humidity and low-humidity conditions did not evidence the formation of any copper chloride salts.

Acknowledgments Part of this research was funded by the Italian Ministry of Instruction, University and Research (MIUR) through the PRIN 2008 project (2008ZRSHHB) and by the European project CHARISMA, FP7 INFRASTRUCTURE no. 228330

\section{References}

1. Plesters J (1956) Stud Conserv 2:110-157

2. Allen J (1992) Vibr Spectr 3:217-237

3. Mazzeo R, Joseph E, Prati S, Millemaggi A (2007) Anal Chim Acta 599:107-117
4. Van der Weerd J, Heeren RMA, Boon JJ (2004) Stud Conserv 49:193-210

5. Tsang JS, Cunningham RH (1991) J Am Inst Conserv 30:163-177

6. Cotte M, Checroun E, Mazel V, Solé A, Richardin P, Taniguchi Y, Walter P, Susini J (2009) e-Pres Sci 6:1-9

7. Echard JP, Bertrand L, von Bohlen A, Le Hô AS, Paris C, Bellot-Gurlet L, Soulier B, Lattuati-Derieux A, Thao S, Robinet L, Lavédrine B, Vaiedelich S (2010) Angew Chem Int Ed 49:197-201

8. Derrick M, Souza L, Kieslick T, Florsheim H, Stulik D (1994) J Am Inst Conserv 33:227-245

9. de Fonjaudran CM, Nevin A, Piqué F, Cather S (2008) Anal Bioanal Chem 392:77-86

10. Pilc J, White R (1995) Natl Gallery Tech Bull 16:73-84

11. Prati S, Joseph E, Sciutto G, Mazzeo R (2010) Acc Chem Res 43:792-801

12. Langley A, Burnstock A (1999) In: Bridgland J (ed) ICOM Committee for Conservation, 12th triennial meeting Lyon 29th August3rd September 1999, vol I. James \& James (Sciences Publishers) Ltd, London, pp 234-241

13. Boon JJ, Asahina S (2006) Microsc Microanal 12:1322-1323

14. van Loon A, Keune K, Boon JJ (2005) In: Art'05 - 8th international conference on the non destructive investigations and microanalysis for the diagnostic and conservation of the cultural and environmental heritage, Lecce

15. Jäegers E, Jäegers E (1999) Brit Mus Occas Pap 135:37-42

16. Prati S, Rosi F, Sciutto G, Mazzeo R, Magrini D, Sotiropoulou S, Van Bos M (2012) Microsc J 103:79-89

17. Cotte M, Dumas P, Taniguchi Y, Checroun E, Walter P, Susini J (2009) C R Phys 10:590-600

18. Van Loon A (2008) Colour changes and chemical reactivity in seventeenth-century oil paintings. PhD thesis, Archetype Publications, London, pp 24-42

19. Thompson DV Jr (1954) Il libro dell'arte, the craftsman's handbook of Cennino d'Andrea Cennini. Dover, New York

20. Kovala-Demertzi D, Papathanasis L, Mazzeo R, Demertzis MA, Varella EA, Prati S (2012) J Cult Herit 13:107-113 